\section{Leinsamen gegen Bluthochdruck}

Iـ Das können manche Hochdruckmittel nicht toppen: Bei hypertensiven pAVK-Patienten, die 6 Monate lang täglich $30 \mathrm{~g}$ gemahlene Leinsamen zu sich nahmen, sank der systolische Blutdruck um $15 \mathrm{mmHg}$ und der diastolische um $8 \mathrm{mmHg}$. In der PlaceboGruppe hingegen war ein leichter Anstieg der Blutdruckwerte zu verzeichnen. So zeigen die Ergebnisse der FlaxPAD-Studie den stärksten antihypertensiven Effekt, der je durch eine diätetische Intervention vermittelt wurde, erklären die Autoren. Verantwortlich für diese Wirkung waren nach ihrer Auskunft die deutlichen Anstiege der Werte für Alfa-Linolensäure, einer protektiven Fettsäure, sowie für Enterolakton, einem potenten Antioxidans. Das Ausmaß der Blutdrucksenkung lässt eine Halbierung der Schlaganfall- rate und eine Senkung des Herzinfarktrisikos um ein Drittel erwarten, schreiben die Autoren des St. Boniface Hospital in Winnipeg.

\section{Lipidsenkende Tomaten}

Iـ Die Story ist schnell erzählt: Forschern der UCLA in Los Angeles ist es gelungen, Tomaten zu züchten, die $6 \mathrm{~F}$ produzieren: ein kleines Lipidmolekül, welches die Wirkung von Apo A-1 kopiert, dem Kernbestandteil der HDL-Cholesterinfraktion. Nach Verzehr der gefriergetrockneten, genetisch veränderten Tomaten sprachen die Probanden umgehend auf die Diät an: Entzündungsparameter sanken, die Konzentration antioxidativer Enzyme stieg an, ebenso das HDL. Die Arteriosklerose Progression verlangsamte sich. Bei den Probanden handelte es sich allerdings vorläufig noch um Mäuse.

\section{Winterzeit ist Herztodzeit}

Nun ja, das wussten wir schon: Jeden Winter sterben die Leute beim Schneeschippen wie die Fliegen. Die Anstrengung in der Kälte. Doch nein: Die Kälte ist es gar nicht, berichten jetzt amerikanische Autoren: Die Herztodrate steigt im Winter an, und zwar um 26 bis 36\%, egal, ob die Leute im sonnigen Kalifornien, Texas oder Florida residieren, oder aber im winterlichen Washington oder Massachusetts.

Ihr Tipp: Auch im Winter eisern einen Lebensstil mit Diät und Bewegung pflegen, der das Herz auf Trab hält.

Dr. med. Dirk Einecke II

IDQuelle: springermedizin.de basierend auf: Jahrestagung der American Heart Association (AHA) vom 3.-7.11.2012 in Los Angeles.

\title{
Thrombozytenaggregationshemmung
}

\section{ASS vor Operationen nicht absetzen}

Ein Patient mit einer dualen Thrombozytenaggregationshemmung muss operiert werden - sollten die Plättchenaggregationshemmer kurzfristig abgesetzt werden? Und falls ja welche?

Nach den offiziellen Leitlinien sollten alle Patienten mit akutem Koronarsyndrom (ACS) eine duale Thrombozytenaggregationshemmung mit ASS plus Clopidogrel, Prasugrel oder Ticagrelor 12 Monate lang erhalten. Das gilt auch für Patienten, die bei einer Koronarintervention einen medikamentös beschichteten Stent bekommen. Im Falle eines Metallstents kann die Plättchenaggregationshemmung bereits nach 4-6 Wochen auf eine Monotherapie mit ASS umgestellt werden. „Die Empfehlungen sollte man konsequent umsetzen, da $30-40 \%$ der StentThrombosen durch Absetzen eines Thrombozytenaggregationshemmers verursacht werden", sagte Prof. Siegfried Rübenacker, Chefarzt der Chirurgischen Klinik in Langenau. Da immer mehr Patienten eine solche Therapie erhielten, werde auch der Chirurg

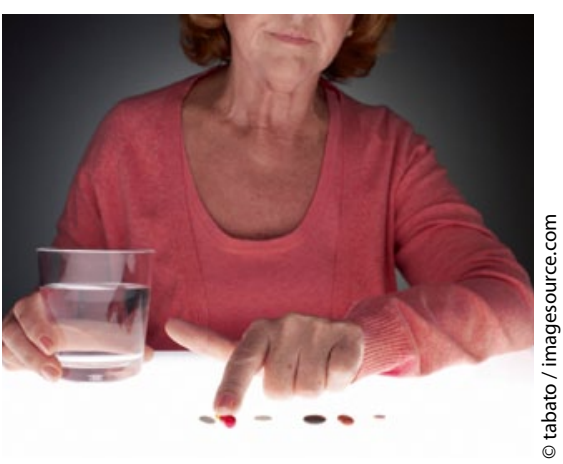

Welchen Thromozytenaggregationshemmer sollte ein Patient vor einer OP noch einnehmen?

zunehmend damit konfrontiert, wie er im Falle eines operativen Eingriffs handeln solle.

\section{ASS wegzulassen erhöht Risiko für ACS}

Unbestritten sollte eine Plättchenaggregationshemmung im Rahmen einer Primärprävention immer 7-12 Tage vor der geplanten Operation abgesetzt werden. Das ist für die Patienten unbedenklich, zumal eine solche Primärprävention nicht evidenzbasiert ist.
Die ASS-Therapie kann frühestens 24 Stunden nach dem Eingriff fortgesetzt werden.

Doch ASS abzusetzen, ist für Patienten mit manifester KHK nicht unproblematisch. Denn Studien zufolge nimmt dann das postoperative Risiko für ein ACS um 10\% zu. Andererseits erhöht ASS aber das Blutungsrisiko um etwa $50 \%$, vorrangig jedoch nur bei intrakraniellen und transurethralen Eingriffen.,Wägt man Nutzen und Risiko ab, sollten Patienten mit ACS bzw. Stentimplantation ASS vor der OP nicht absetzen“, rät Rübenacker. Soweit möglich, sollte die OP vor allem bei Patienten mit einem Stent so lange verschoben werden, bis eine duale Plättchenaggregationshemmung nicht mehr nötig ist. Ansonsten sollte der zweite Plättchenaggregationshemmer vorübergehend abgesetzt und perioperativ nur ASS gegeben werden.

Dr. med. Peter Stiefelhagen II

IDQuelle: 11. Expertentreffen: Niedermolekulare Heparine - up to date 2012, 13.10.2012 in Berlin 